\title{
A NONLINEAR PARABOLIC SYSTEM IN THE THEORY OF COMBUSTION*
}

\author{
BY
}

D. H. SATTINGER (University of Minnesota)

1. Introduction. Let $T$ be the temperature and $n$ the concentration of a combustible substance. As a simple model governing the combustion of the material we consider the following system of equations:

$$
\begin{gathered}
K_{1} \Delta T+Q n \exp (-E / R T)-\frac{\partial T}{\partial t}=0 \\
K_{2} \Delta n-n \exp (-E / R T)-\frac{\partial n}{\partial t}=0
\end{gathered}
$$

where the constant $Q$ is the heat of reaction; the constants $K_{1}$ and $K_{2}$ are the thermal and material diffusion coefficients; the term $\exp (-E / R T)$ is the Arrenhius rate factor; $E$ is the activation energy; and $R$ is the universal gas constant. Eq. (1.1) are considered on a bounded domain $D$ with boundary conditions

$$
T=T_{0}, \partial n / \partial \nu=0 \text { on } \partial D
$$

together with initial conditions

$$
T(x, 0)=T_{0}(x), \quad n(x, 0)=n_{0}(x) .
$$

We shall assume throughout this paper that $T_{0}(x) \geq T_{0}$.

The boundary-initial value problem (1.1)-(1.3) constitutes only the simplest model for combustion problems. A discussion of the derivation of the general equations of chemical kinetics may be found in the book by Gavalas [7]. The particular model (1.1)(1.3) has been discussed in an article by Gel'fand [6], and in [1].

As we see in Sec. 2, the solution $T(x, t), n(x, t)$ of the boundary-initial value problem (1.1)-(1.3) tends asymptotically to the unique steady state $T(x) \equiv T_{0}, n(x) \equiv 0$, and this ultimate state is independent of the initial data. The interest in the problem lies not in the final state which the system eventually reaches, but rather the manner in which that state is attained. In fact, the initial behavior of the system can differ markedly, depending on the initial data.

Let $\alpha=E / R T_{0}$ and put $\epsilon=\exp (-\alpha)$. In actual problems $\alpha$ typically may range from 20 to 100 [8], and so we shall assume in this article that $\epsilon$ is a small parameter (though not necessarily so small as exp (-20)). If the initial temperature and concentration are "below criticality", the combustion proceeds very slowly, on a time scale of order $\epsilon$, with the temperature remaining relatively close to $T_{0}$, and the temperature and concentration decaying as $\exp (-\epsilon t)$. On the other hand, if the temperature and

* Received July 10, 1973; revised version received October 17, 1973. This research was supported in part by the National Science Foundation under research grant GP-37512. 
concentration are initially above "criticality" the reaction proceeds very quickly, with a rapid build-up to a high temperature, and a rapid burn-up of the reactant.

The two different processes are, of course, well known in the theory of chemical reactions (see, e.g., [1]). The first case, that of slow combustion, has been discussed qualitatively by Gel'fand [6]. He states "In [this] type of reaction, in the case of sufficiently small $\epsilon$, one can on a certain interval $\left(0,2 t_{0}\right)$ neglect the burning up of the combustible component, i.e. one can assume $n \equiv n_{0} \equiv$ const., where $n_{0}$ is the initial concentration of the combustible component. Substituting this relation in [the equation for $T$ ], we find that on the interval of time $\left(0, t_{0}\right)$ the temperature $T$ will be determined from the equation

$$
\partial T / \partial t=K_{1} \Delta T+Q n_{0} \exp (-E / R T)^{\prime \prime} .
$$

Thus, by this heuristic argument, the study of the behavior of the system (1.1) is reduced to a study of the single equation for the temperature.

In Sec. 7 we construct, by a perturbation argument, functions which approximate the solutions of the system (1.1)-(1.3) uniformly on $0<t<\infty$ to order $O(\epsilon \exp (-\epsilon t))$. These approximations exhibit clearly the type of behavior described by Gel'fand. The concentration $n_{0}$ decays very slowly $\left(n_{0} \sim \exp (-\epsilon t)\right)$ and so, to a first approximation, $T$ tends to an equilibrium solution of (1.4), viz.

$$
K_{1} \Delta T+Q n_{0}(\epsilon t) \exp (-E / R T)=0 .
$$

Such a slowly-varying solution is called a metastable state or a quasistatic state. The perturbation arguments are of a type sometimes called a "two-time perturbation method". The two time scales involved are the initial build-up time, which is fairly rapid, and the long-time, quasi-static behavior, of order $\epsilon$. When the initial data is below criticality the system adjusts, on a time scale of order one, to the metastable state, and then decays very slowly on a time scale of order $\epsilon t$. Related perturbation techniques have been developed by Fife [5] for linear parabolic systems, and by Hoppensteadt [2] for a single quasilinear parabolic equation.

We have not been able to construct an accurate approximation to the solution of Eq. (1.1) in the "supercritical" case. There are, nevertheless, a few comments worth making. Consider the following system of ordinary differential equations, which constitutes an even simpler mathematical model of the combustion problem:

$$
d \theta / d t=-\theta+H n f(\theta), \quad d n / d t=-\epsilon n f(\theta)
$$

where $f(\theta)=\exp \left\{\theta /\left(1+\theta \alpha^{-1}\right)\right\}$ and $\epsilon=\exp (-\alpha)$.

These equations are obtained in the following way: the equations are first transformed to the form (4.1), (4.2) (see Sec. 4). In Eq. (4.1) the Laplacian combined with the boundary condition $\theta=0$ produces a dissipative effect. Therefore, in going to the system of ordinary differential equations the term $\Delta \theta$ is replaced by the dissipative term $-\theta$. In the equation for the concentration there is no dissipative effect, due to the boundary condition $\partial n / \partial \nu=0$, and the only mechanism which causes $n$ to decrease is the combustion process itself. Therefore the term $\Delta n$ is dropped altogether. The resulting equations exhibit qualitative features similar to the original system of partial differential equations. For proper ranges of the parameters, the steady-state equation for the temperature with fixed concentration has three solutions.

In particular, the same criticality feature is present. The ordinary differential equa- 
tions are easily treated on a computer. It was found that, if the initial data is slightly above criticality, the temperature rises slowly for a period of time until the system enters a regime in which the temperature rises very rapidly. The numerical computations we made, showing a sudden and very rapid increase in temperature, suggest that in the supercritical case there is a rapid time scale involved (the explosion); but we have not succeeded in constructing an adequate perturbation scheme to describe this case, and the present paper is confined to a discussion of the singular perturbation argument in the slow combustion case. The above system of equations is closely related to the equation of a tubular reactor (see [14], p. 286; put $\xi=1-n$ ). Aris' book contains a qualitative description of the behavior of solutions of the system of differential equations. The graph on p. 287 shows explicitly the phenomena of sub- and supercriticality. We should also like to refer the reader to the paper [15].

The methods used in this paper for establishing the validity of the perturbation arguments are based on maximum principle arguments. A similar technique was used in [12] to justify singular perturbation arguments for single elliptic equations. The procedure there was to modify slightly the perturbation calculations to construct functions which satisfied appropriate differential inequalities, thereby obtaining upper and lower bounds on the actual solution. The problems considered there were nonlinear elliptic boundary-value problems. The problem under consideration here is a nonlinear parabolic system. Although the details of the argument are somewhat more complicated, the basic principles are the same-to modify the perturbation arguments in an appropriate way, thereby constructing functions which satisfy a system of differential inequalities.

In Sec. 2 we show that for arbitrary initial data, the solutions of (1.1)-(1.3) tend as $t \rightarrow \infty$ to the unique equilibrium state $T \equiv T_{0}, n \equiv 0$. In Sec. 3 we prove a comparison theorem for the system, based on the maximum principle. In Sec. 4 we review the theory of multiple stationary states of the temperature equation with $n=n_{0}=$ const., viz.

$$
K_{1} \Delta T+Q n_{0} \exp (-E / R T)=0,\left.\quad T\right|_{\partial D}=T_{0} .
$$

In Sec. 5 we construct asymptotic series for the metastable states. The validity of these series is established in Sec. 6. Finally, in Sec. 7 we construct an approximate solution uniformly valid to order $\epsilon \exp (-\epsilon t)$ on $0<t<\infty$ and establish its validity.

The author would like to acknowledge several useful discussions with Professor D. G. Aronson, who made a number of useful comments regarding the application of the maximum principle to the system (1.1)-(1.3).

Throughout this paper $D$ denotes a smoothly bounded domain in $\mathbf{R}^{n}$ and $x$ denotes a point in $D$. From now on, for convenience, we take both diffusion constants to be unity, since their values do not play a critical role in our analysis.

\section{Asymptotic behavior.}

Theorem 2.1. For arbitrary bounded initial data the solutions of (1.1)-(1.3) tend, as $t \rightarrow \infty$, to $T=T_{0}, n=0$. This is thus the unique steady state.

Proof: From the temperature equation we have

$$
\Delta T-(\partial T / \partial t) \leq 0, T=T_{0} \text { on } \partial D
$$

and so $T>T_{0}$ in $D \times(0, \infty)$ by the maximum principle. The concentration $n(x, t)$ therefore satisfies the differential inequality 


$$
\Delta n-\frac{\partial n}{\partial t}-n \exp \left(-E / R T_{0}\right) \geq 0,
$$

since $\exp (-E / R T)$ is a monotone function of $T$. Letting $N=\max n(x, 0)$, consider the function $n(t)=N \exp (-\epsilon t)$ where $\epsilon=\exp \left(-E / R T_{0}\right)$. This function satisfies

$$
\Delta n-\partial n / \partial t-n \epsilon=0, \quad \partial n / \partial \nu=0 \quad \text { on } \quad \partial D
$$

and so, by the comparison theorem for parabolic equations ([11], p. 187), $n(x, t) \leq n(t)$. Returning to Eq. (1.1), we get the following parabolic inequality for $T$ :

$$
\Delta T+Q N \exp (-\epsilon t)-\partial T / \partial t \geq \Delta T+Q n \exp (-E / R T)-\partial T / \partial t=0 .
$$

Therefore $T(x, t) \leq \hat{T}(x, t)$, where $\hat{T}$ satisfies

$$
\Delta \hat{T}+Q N \exp (-\epsilon t)-\partial \hat{T} / \partial t=0, \hat{T}(x, 0) \geq T(x, 0),\left.\hat{T}\right|_{\partial D}=T_{0} .
$$

On the other hand, it is easily seen that $\hat{T}(x, t) \rightarrow T_{0}$ as $t \rightarrow \infty$. This completes the proof of Theorem (1.1).

3. A comparison theorem. The theorem below is useful in analyzing the behavior of solutions of the initial-value problem (1.1)-(1.3). It is the analogue of the comparison theorem for a single parabolic equation (see Protter and Weinberger [11], p. 187). To make things a little more general we consider the following boundary-initial value problem:

$$
\begin{gathered}
\Delta T+f(T, n)-\partial T / \partial t=0, \quad \Delta n+g(T, n)-\partial n / \partial t=0 \\
T(x, 0)=\alpha(x) \quad n(x, 0)=\beta(x) \\
\left.T\right|_{\partial D}=\left.T_{0} \quad \frac{\partial n}{\partial \nu}\right|_{\partial D}=0
\end{gathered}
$$

where $f$ is an increasing and $g$ is a decreasing function of each of its variables.

Theorem 3.1. Suppose there are functions $n_{1}, n_{2}, T_{1}$, and $T_{2}$ defined on $\bar{D} \times$ $\left[0, t_{0}\right)$ which are $C^{2}$ in $x, C^{1}$ in $t$, and which satisfy the following conditions:

(i) $n_{1}<n_{2}, T_{1}<T_{2}$.

(ii) $T_{1} \leq T_{0} \leq T_{2}$ and $\partial n_{1} / \partial \nu \leq 0 \leq \partial n_{2} / \partial \nu$ on $\partial D$;

$T_{1}(x, 0)<\alpha(x)<T_{2}(x, 0)$ and $n_{1}(x, 0)<\beta(x)<n_{2}(x, 0)$.

(iii) $\Delta T_{2}+f\left(T_{2}, n_{2}\right)-\partial T_{2} / \partial t \leq 0$, $\Delta n_{1}+g\left(T_{2}, n_{1}\right)-\partial n_{1} / \partial t \geq 0$ $\Delta T_{1}+f\left(T_{1}, n_{1}\right)-\partial T_{1} / \partial t \geq 0$

Then

$$
\Delta n_{2}+g\left(T_{1}, n_{2}\right)-\partial n_{2} / \partial t \leq 0 \text {. }
$$

$$
T_{1}(x, t)<T(x, t)<T_{2}(x, t), \quad n_{1}(x, t)<n(x, t)<n_{2}(x, t)
$$

on $D \times\left[0, t_{0}\right)$. (Equality may hold on the boundary.)

Proof: Let $I$ be the set of $t \geq 0$ for which the (strict) inequalities (3.4) are valid in the interior of $D$. From (ii) $t=0$ belongs to $I$. By continuity of the functions $n$ and $T$, $I$ is open. Let us show also that $I$ is closed. Suppose, then, that $\left[0, t_{1}\right)$ is contained in $I$ and let us show that $t_{1} \in I$. 
By continuity we have

$$
T_{1}(x, t) \leq T(x, t) \leq T_{2}(x, t), \quad n_{1}(x, t) \leq n(x, t) \leq n_{2}(x, t)
$$

on $\bar{D} \times\left[0, t_{1}\right]$. Since $n \leq n_{2}$ we have

$$
0=\Delta T+f(T, n)-\partial T / \partial t \leq \Delta T+f\left(T, n_{2}\right)-\partial T / \partial t
$$

while

$$
\Delta T_{2}+f\left(T_{2}, n_{2}\right)-\frac{\partial T_{2}}{\partial t} \leq 0 .
$$

Subtracting these two inequalities and putting $w=T-T_{2}$, we get

$$
\Delta w+\hat{f} w-\frac{\partial w}{\partial t} \geq 0
$$

where

$$
\hat{f}=\int_{0}^{1} \frac{\partial f}{\partial T}\left(T_{2}+s\left(T-T_{2}\right), n\right) d s .
$$

Now $w(x, 0)<0$ and $w(x, t) \leq 0$ on $\partial D$. Therefore, by the strong maximum principle, either $w(x, t) \equiv 0$ in $\bar{D} \times\left[0, t_{1}\right)$, which is a contradiction, or $w\left(x, t_{1}\right)<0$ when $x \in D$.

By a similar argument we obtain for $w=n-n_{2}$ the differential inequalities

$$
\Delta w+g w-\partial w / \partial t \geq 0, \quad \partial w / \partial \nu \leq 0 \text { on } \partial D, \quad w(x, 0)<0
$$

where

$$
\hat{g}=\int_{0}^{1} \frac{\partial g}{\partial c}\left(T_{1}, n_{2}+s\left(n-n_{2}\right)\right) d s .
$$

Again, by the strong maximum principle, since $w$ is not identically zero on $D \times\left[0, t_{1}\right)$, $w\left(x, t_{1}\right)<0$ for $x \in D$. The other two inequalities follow by similar arguments; and so we see that $t_{1} \in I$ as well, so that $I$ is closed.

This completes the proof of the comparison theorem.

4. Stationary solutions of the first equation. We begin by introducing some new variables, as in [8]. We let

$$
T=T_{0}+\left(R T_{0}{ }^{2} / E\right) \theta, \quad \alpha=E / R T_{0}, \quad H=\left(Q E / R T_{0}{ }^{2}\right) \exp (-\alpha) .
$$

Then Eq. (1.1)-(1.3) take the form

$$
\begin{gathered}
\Delta \theta H n f(\theta)-\partial \theta / \partial t=0 \\
\Delta n-n \epsilon f(\theta)-\partial n / \partial t=0, \quad \partial n / \partial \nu=\theta=0 \quad \text { on } \quad \partial D,
\end{gathered}
$$

where

$$
f(\theta)=\exp \left\{\frac{\theta}{1+\theta / \alpha}\right\} .
$$

As mentioned in the introduction, we take $\epsilon=\exp (-\alpha)$ to be a small parameter, but we do not assume that $H$ is small. 
If initially $\theta$ is small and remains small, then

$$
\Delta n-\partial n / \partial t=O(\epsilon)
$$

and if we simply set the right side equal to zero, then $n(x, t)$ tends asymptotically to

$$
\bar{n}=\frac{1}{\operatorname{meas} D} \iint_{D} n(x, 0) d x .
$$

According to Gel'fand's argument, we assume $n(x, t) \equiv \bar{n}$ and that $\theta$ is governed by

$$
\Delta \theta+\lambda f(\theta)-\partial \theta / \partial t=0
$$

where $\lambda=H \bar{n}$. In order to understand the behavior of solutions of (4.3) it is instructive to look at the associated time-independent equations

$$
\Delta \theta+\lambda f(\theta)=0,\left.\quad \theta\right|_{\partial D}=0 .
$$

Eq. (4.4) has one, two, or three solutions, depending on the value of $\lambda$. (See [8], [10] where the boundary-value problem is analyzed on a spherical domain.)

One of the concerns of this paper is the relevance of the solutions of (4.4) to the initialvalue problem (4.1)-(4.2). It will therefore be helpful if we review briefly the structure of solutions of (4.4).

Parks [8] has found numerically that on a spherical domain (4.4) has the structure of spherically symmetric solutions shown qualitatively in Fig. 1. For $\lambda\rangle \lambda_{2}$ or $\lambda\left\langle\lambda_{1}\right.$ there is only one equilibrium solution, while for $\lambda_{1}<\lambda<\lambda_{2}$ there are three solutions, which we may denote by $w_{i}(x, \lambda), i=1,2,3$. We have $w_{1}<w_{2}<w_{3}$, with $w_{1}$ and $w_{3}$ stable equilibria (relative to (4.3)) and $w_{2}$ unstable. As $\lambda \rightarrow \lambda_{2}$ the solutions $w_{1}$ and $w_{2}$ coalesce and then disappear for $\lambda>\lambda_{2}$. Similarly, $w_{2}$ and $w_{3}$ coalesce and disappear as $\lambda$ passes through $\lambda_{1}$.

(Remark. The instability of the middle solution can be proved by a degree argument, knowing the stability of the top or bottom solution. See [13].)

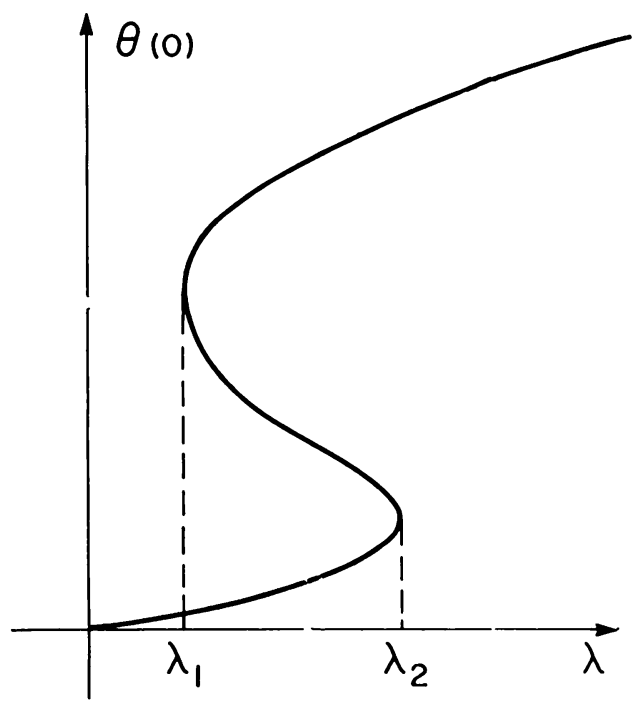

Fig. 1. 
Some rough estimates of the three solutions can be obtained as follows. For convenience, let us rewrite (4.4) in the form

$$
\Delta u+\lambda \exp (-1 / u)=0,\left.\quad u\right|_{\partial D}=1 / \alpha
$$

where

$$
u=R T / E=\frac{1}{\alpha}+\frac{\theta}{\alpha^{2}} .
$$

Let $u(0)=a$; then $u^{\prime}(0)=0$, since $u$ is spherically symmetric, and near $r=0 u$ is given approximately by

$$
u(r, a)=a-\frac{\lambda}{6} \exp (-1 / \alpha) r^{2} .
$$

Though this approximation is accurate only near the origin, we nevertheless assume it out to the boundary $r=1$ and apply the boundary condition there. We then obtain the transcendental equation

$$
u(1, a)=a-\frac{\lambda}{b} \exp (-1 / a)=\frac{1}{\alpha} .
$$

Depending on $\lambda$, this equation has one, two, or three roots, as indicated in Fig. 2. For large $\alpha$ the smallest root $a_{1}$ is approximately $1 / \alpha$. For $a_{1}<a<a_{2}$ or $a_{3}<a$ the function in (4.6) is an upper solution for the boundary-value problem (4.4), i.e.

$$
\Delta u+\lambda \exp (-1 / u) \leq 0,\left.\quad u\right|_{\partial D} \geq 1 / \alpha .
$$

(The reader may easily check these inequalities.) Thus $u(r, a)$ for $a=a_{1}$ or $a=a_{3}$

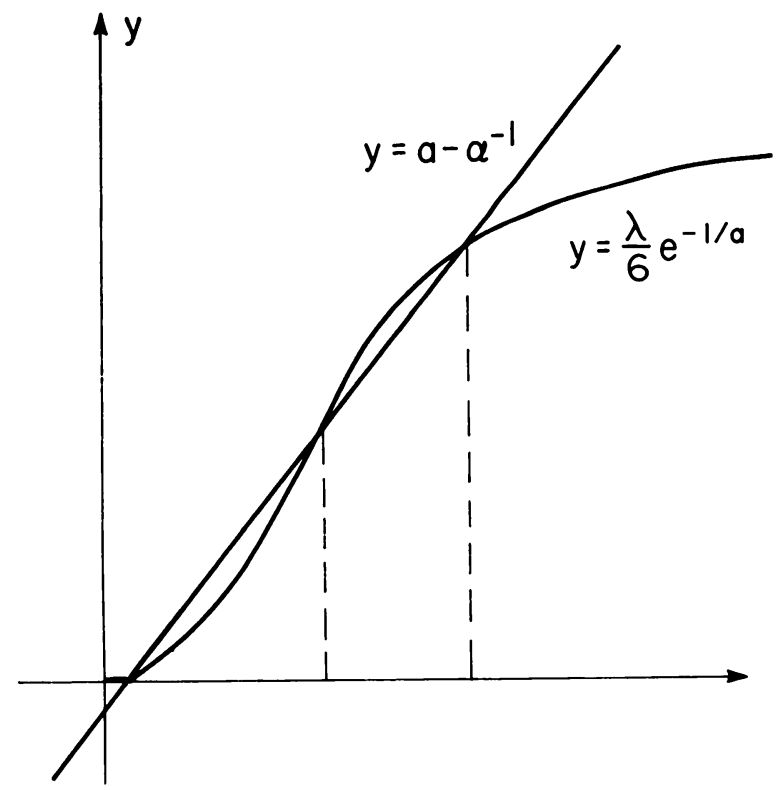

FIG. 2. 
provides an upper estimate, respectively, of $\theta_{1}$ or $\theta_{3}$ (see [12]). For $a=a_{2}$ the function $u\left(r, a_{2}\right)$ provides a lower estimate of $\theta_{2}$.

On the other hand, the function

$$
v(r, \alpha)=\frac{1}{\alpha}+\frac{\lambda}{6} \exp (-\alpha)\left(1-r^{2}\right)
$$

is a lower solution:

$$
\Delta v+\lambda \exp (-1 / v) \geq 0, \quad v(1)=1 / \alpha .
$$

Furthermore, since

$$
a_{1}=\frac{1}{\alpha}+\frac{\lambda}{6} \exp \left(-1 / a_{1}\right)>1 / \alpha,
$$

$v(r, \alpha) \leq u\left(r, a_{1}\right)$. Therefore $v(r, \alpha) \leq \theta_{1} \leq u\left(r, a_{1}\right)$.

For a more extensive discussion of the solutions of (4.4) the reader is referred to the article by Parter [10]. Finally, we recall [12] that if $0<\theta(x, 0)<w_{2}(x, \lambda)$ then $\theta(x, t) \rightarrow$ $w_{1}(x, \lambda)$ as $t \rightarrow \infty$, where $\theta(x, t)$ is the solution to the initial-value problem (4.3) with initial data $\theta(x, 0)$. Similarly, $\theta(x, t) \rightarrow w_{3}(x, \lambda)$ if $\theta(x, 0)>w_{2}(x)$.

The exponential approximation. If $\theta / \alpha \ll 1$ we can approximate $f(\theta)$ by

$$
f(\theta)=\exp \left\{\frac{\theta}{1+\theta / \alpha}\right\} \approx e^{\theta}
$$

and this leads to the so-called exponential approximation in chemical kinetics (see [1], [6]). The boundary-value problem (4.4) is then replaced by

$$
\Delta \theta+\lambda \exp (\theta)=0,\left.\quad \theta\right|_{\partial D}=0,
$$

a problem which was analyzed extensively by Gel'fand [6]. For $\lambda$ less than a certain critical value (4.7) has a (small) stable solution, say $\bar{\theta}$. Since $\exp (\theta) \geq \exp (\theta / 1+\theta / \alpha)$, we see that $\bar{\theta}$ is an upper solution of (4.4). Thus the critical value obtained by solving (4.7) gives a lower bound for the critical value of (4.4).

5. Metastable states; asymptotic series. As we stated in the introduction, if the initial temperature and concentration are below criticality, the combustion proceeds very slowly, with quantities varying slowly, on a time scale of order $\epsilon=\exp (-\alpha)$. With reference to Sec. 4, we may assert that the system is subcritical if $\theta(x, 0)<w_{2}(x, H N)$, where $N=\sup n(x, 0)$. For then we can show that $\theta(x, t)<w_{2}(x, H N)$ for all $t$. In fact, referring to the notation in Theorem 3.1, we may take $n_{1}=0, n_{2}=N, \theta_{1}=0$ and $\theta_{2}(x, t)$ to be the solution of

$$
\Delta \theta_{2}+H N f\left(\theta_{2}\right)-\partial \theta_{2} / \partial t=0 .
$$

with the initial conditions for $\theta_{2}$ chosen so that $\theta(x, 0)<\theta_{2}(x, 0)$. Now

$$
\Delta w_{2}+H N f\left(w_{2}\right)-\partial w_{2} / \partial t=0
$$

while $\theta_{2}=w_{2}$ on $\partial D$ and $\theta_{2}(x, 0)<w_{2}(x, H N)$. It therefore follows from the comparison theorem for parabolic equations ([11], p. 187) that $\theta_{2}(x, t)<w_{2}(x, H N)$.

In this section we consider a specific class of solutions of (4.1)-(4.2)-the metastable 
states. Specifically, consider a solution $\theta(x, t), n(x, t)$ of $(4.1),(4.2)$, where

$$
\begin{gathered}
n(x, 0) \equiv c_{0}=\text { const. } \\
H c_{0}<\lambda_{2}, \\
\theta(x, 0)=w_{1}\left(x, H c_{0}\right) .
\end{gathered}
$$

(The terms $\lambda_{2}$ and $w_{1}(x, \lambda)$ were introduced in Sec. 4.) We shall see below that such a solution depends only on $x$ and $\tau=\epsilon t$; it will be called a metastable state.

In this section we develop a formal asymptotic series approximating such a solution. The validity of the series is demonstrated in Sec. 6 .

Putting $\tau=\epsilon t$, we obtain the following form of Eqs. (4.1), (4.2):

$$
\Delta \theta+H n f(\theta)=\epsilon \frac{\partial \theta}{\partial \tau}, \quad \Delta n=\epsilon\left[\frac{\partial n}{\partial \tau}+n f(\theta)\right] .
$$

We assume formal expansions of $\theta$ and $n$ :

$$
\theta=\theta_{0}+\epsilon \theta_{1}+\cdots, \quad n=n_{0}+\epsilon n_{1}+\cdots .
$$

Substituting these expansions into (5.4), we get at the first stage

$$
\Delta \theta_{0}+H n_{0} f\left(\theta_{0}\right)=0, \quad \Delta n_{0}=0, \quad \partial n_{0} / \partial \nu=\theta_{0}=0 \quad \text { on } \quad \partial D .
$$

From these equations it is evident that $n_{0}$ is independent of the spatial variables; thus $n_{0}=n_{0}(\tau)$ and $\theta_{0}$ satisfies

$$
\Delta \theta_{0}+H n_{0} f\left(\theta_{0}\right)=0 .
$$

We choose the minimal positive solution here; that is, $\theta_{0}=w_{1}\left(x, H n_{0}\right)$. Note that this solution is stable; that is, all eigenvalues of the derived operator

$$
L \varphi=\Delta \varphi+H n_{0} f^{\prime}\left(\theta_{0}\right) \varphi
$$

are negative. We shall use this fact later on. We determine $n_{0}$ at the next stage as follows. The equation for $n_{1}$ is

$$
\Delta n_{1}=\left(d n_{0} / d \tau\right)+n_{0} f\left(\theta_{0}\right), \quad \partial n_{1} / \partial v=0 .
$$

The solvability condition for this Neumann problem is

$$
\left(d n_{0} / d \tau\right)+n_{0} \overline{f\left(\theta_{0}\right)}=0
$$

where, for any function $g$ defined on $D$,

$$
\bar{g}=\frac{1}{\operatorname{meas} D} \iint_{D} g(x) d x .
$$

Eqs. (5.5) and (5.7) constitute a coupled system for $n_{0}$ and $\theta_{0}$. Since $f(\theta) \geq 1$ we deduce from (5.7) that $n_{0}(\tau) \leq c_{0} \exp (-\tau)$. It then follows from (5.5) that $\theta_{0}=O(\exp (-\tau))$ also.

Remark: Although the system (5.5)-(5.7) is coupled in a slightly unusual manner, there is no difficulty in proving the existence of a solution. If we denote the solution of

$$
\Delta \theta_{0}+\lambda f\left(\theta_{0}\right)=0, \quad \theta=0 \text { on } \partial D
$$

by $\theta_{0}(x, \lambda)$, then $\theta_{0}$ depends differentiably on $\lambda$ in any interval excluding the critical 
points $\lambda_{1}, \lambda_{2}$. This is a simple consequence of the implicit function theorem on a Banach space. Therefore, in (5.7),

$$
\overline{f\left(\theta_{0}\left(x, H n_{0}\right)\right)}=\frac{1}{\text { meas } D} \iint_{D} f\left(\theta_{0}\left(x, H n_{0}\right)\right) d x
$$

is a $C^{1}$ function of $n_{0}$. The classical existence theorems thus apply to the ordinary differential equation (5.7). Once $n_{0}(\tau)$ is determined from (5.7) we may substitute that function in (5.5) and solve for $\theta_{0}=\theta_{0}(x, \tau)$. (We regard $\tau$ as a parameter.)

If $\hat{n}_{1}$ denotes the unique solution of (5.6) for which $\overline{\hat{n}}_{1}=0$, then the general solution of (5.6) is of the form

$$
n_{1}=\hat{n}_{1}+\xi_{1}(\tau)
$$

where the function $\xi_{1}(\tau)$ is to be determined at the next stage.

Now suppose we have constructed functions $\theta_{0}, \cdots \theta_{l-1}, n_{0}, \cdots n_{l-1}$, and $\hat{n}_{l}$ such that

$$
T_{l-1}=\sum_{k=0}^{l-1} \epsilon^{k} \theta_{k}, \quad C_{l}=\sum_{k=0}^{l-1} \epsilon^{k} n_{k}+\epsilon^{l} \hat{n}_{l}
$$

satisfy Eqs. (5.4) to order $\epsilon^{l}$ and $\epsilon^{l+1}$ respectively. That is,

$$
\begin{aligned}
& \Delta T_{l-1}+H C_{l} f\left(T_{l-1}\right)-\epsilon \frac{\partial T_{l-1}}{\partial \tau}=\epsilon^{l} Z_{l}, \\
& \Delta C_{l}-\epsilon\left\{C_{l} f\left(T_{l-1}\right)+\frac{\partial C_{l}}{\partial \tau}\right\}=\epsilon^{l+1} W_{l+1},
\end{aligned}
$$

where $Z_{l}, W_{l+1}$ are functions of $\theta_{0}, \cdots \theta_{l-1}, n_{0}, \cdots n_{l-1}, \hat{n}_{l}, \epsilon$. In order to determine $\xi_{n}, \theta_{l}$, and $\hat{n}_{l+1}$, we substitute

$$
T_{l-1}+\epsilon^{l} \theta_{l}, \text { and } C_{l}+\epsilon^{l} \xi_{l}+\epsilon^{l+1} \hat{n}_{l+1}
$$

into Eqs. (5.4) and require these equations to be satisfied to order $\epsilon^{l+1}$ and $\epsilon^{l+2}$ respectively. This procedure leads, in the obvious way, to the equations

$$
\begin{aligned}
& \Delta \theta_{l}+H n_{0} f^{\prime}\left(\theta_{0}\right) \theta_{l}+\xi_{l} H f\left(\theta_{0}\right)-Z_{l}=0, \\
& \Delta \hat{n}_{l+1}=\frac{d \xi_{l}}{d \tau}+\xi_{l} f\left(\theta_{0}\right)+n_{0} f^{\prime}\left(\theta_{0}\right) \theta_{l}+W_{l+1} .
\end{aligned}
$$

Applying the solvability condition for (5.12), we obtain the equation

$$
\frac{d \xi_{l}}{d \tau}+n_{0} \overline{f^{\prime}\left(\theta_{0}\right) \theta_{l}}+\xi_{l} \overline{f\left(\theta_{0}\right)}+\overline{W_{l+1}} \quad 0 .
$$

Eqs. (5.11) and (5.13) constitute a coupled system for $\theta_{l}$ and $\xi_{l}$. The equation for $\theta_{l}$ is linear, so $\theta_{l}$ has the representation

$$
\theta_{l}=A_{l}+\xi_{l} B_{l}
$$

where

$\Delta A_{l}+H n_{0} f^{\prime}\left(\theta_{0}\right) A_{l}=Z_{l}, \quad \Delta B_{l}+H n_{0} f^{\prime}\left(\theta_{0}\right) B_{l}+H f\left(\theta_{0}\right)=0, \quad A_{l}=B_{l}=0 \quad$ on $\quad \partial D$. These equations are uniquely solvable for $A_{l}$ and $B_{l}$. (The equilibrium solution $\theta_{0}(x, \tau)$ is stable for fixed $\tau$; hence all eigenvalues of the derived operator $L \psi=\Delta \psi+H n_{0} f^{\prime}\left(\theta_{0}\right) \psi$ 
are negative.) Substituting the representation (5.14) for $\theta_{l}$ into (5.13) we obtain the following equation for $\xi_{l}$ :

$$
\begin{aligned}
& \frac{d \xi_{l}}{d \tau}+\xi_{l} \overline{f\left(\theta_{0}\right)}+\xi_{l} n_{0} \overline{f^{\prime}\left(\theta_{0}\right) B_{l}} \\
& +n_{0} \overline{f^{\prime}\left(\theta_{0}\right) A_{l}}+\overline{W_{l+1}}=0 .
\end{aligned}
$$

By induction it may be shown that each of the functions $\theta_{0}, \cdots \theta_{l-1}, n_{0}, \cdots n_{l-1}$ behaves like $\exp (-\tau)$ as $\tau \rightarrow \infty$. Therefore $\overline{W_{l+1}}$ also behaves like exp $(-\tau)$ as $\tau \rightarrow \infty$. Due to the dissipative term, $\xi_{l} \overline{f\left(\theta_{0}\right)}$ in the equation for $\xi_{l}$ and hence also $\theta_{l}$ decay like $\exp (-\tau)$. Having found $\xi_{l}$ we can then find $\hat{n}_{l+1}$, and $\theta_{l}$ is given by (5.14). The construction of the asymptotic series is thus complete.

6. Validity of the asymptotic expansion. We prove in this section that the series constructed in the previous section constitutes a valid asymptotic expansion of the metastable state. Specifically,

Theorem 6.1. Let $\theta(x, t), n(x, t)$ be the solutions of (4-1)-(4.2) satisfying the special initial conditions (5.1)-(5.3). Then

$$
\begin{aligned}
& \theta(x, t)-\sum_{k=0}^{l-1} \epsilon^{k} \theta_{k}(x, \tau)=O\left(\epsilon^{l} \exp (-\tau)\right) \\
& n(x, t)-\sum_{k=0}^{l} \epsilon^{k} n_{k}(x, \tau)=O\left(\epsilon^{l+1} \exp (-\tau)\right)
\end{aligned}
$$

uniformly on $\bar{D} \times(0, \infty)$.

Proof: Let $T_{l}$ and $C_{l}$ be given as in Sec. 5 (the line above (5.8)). We are to show that $T_{l-1}$ and $C_{l}$ are accurate approximations to $\theta(x, t)$ and $n(x, t)$ to order $\epsilon^{l} \exp (-\tau)$. To prove the theorem we shall perturb $T_{l-1}$ and $C_{l}$ by functions of order $\epsilon^{l}$ in such a way as to construct appropriate comparison functions as described in Theorem 3.1. Specifically, we consider functions of the form

$$
T=T_{l-1}+\epsilon^{l} \theta_{l}+\epsilon^{l} U=T_{l}+\epsilon^{l} V
$$

and

$$
\begin{aligned}
C & =C_{l}-\epsilon^{l} n_{l}+\epsilon^{l+1} \hat{n}_{l+1}+\epsilon^{l} V+\epsilon^{l+1} \hat{v} \\
& =C_{l+1}+\epsilon^{l} V+\epsilon^{l+1} \hat{v}
\end{aligned}
$$

where $U=U(x, \tau), V=V(\tau)$, and $v=v(x, \tau)$ are to be determined. Our intention is to construct $U, V$, and $\hat{v}$ so that the four functions

$$
\begin{gathered}
T_{l}+\epsilon^{l} V, \quad T_{l}-\epsilon^{l} V, \\
C_{l+1}+\epsilon^{l} V+\epsilon^{l+1} \hat{v}, \quad C_{l+1}-\epsilon^{l} V-\epsilon^{l+1} \hat{v}
\end{gathered}
$$

satisfy the inequalities of Theorem 3.1 for all sufficiently small (positive) $\epsilon$. Then $\theta(x, t)$ will be bounded as follows:

$$
T_{\imath}-\epsilon^{l} U<\theta<T_{\imath}+\epsilon^{l} U,
$$

and a similar estimate will hold for the concentration. In addition, $U, V$, and $\hat{v}$ will be constructed so that they decay like $\exp (-\tau)$ as $\tau \rightarrow \infty$; so from the above inequality 
we get

$$
\left|\theta(x, t)-T_{l-1}\right|=O\left(\epsilon^{l} l^{-\tau}\right) .
$$

Substituting the above expressions for $T$ and $C$ into Eqs. (5.4), we obtain

$$
\begin{aligned}
& \Delta T+H C f(T)-\frac{\partial T}{\partial t}=\epsilon^{l}\left(\Delta U+H n_{0} f^{\prime}\left(\theta_{0}\right) U+H V f\left(\theta_{0}\right)\right)+O\left(\epsilon^{l+1} \exp (-\tau)\right) \\
& \Delta C-\epsilon C f(T)-\frac{\partial C}{\partial t}=\epsilon^{l+1}\left[\Delta \hat{v}-V f\left(\theta_{0}\right)-n_{0} f^{\prime}\left(\theta_{0}\right) U-\frac{d V}{d \tau}\right]+O\left(\epsilon^{l+2} \exp (-\tau)\right)
\end{aligned}
$$

provided that $U, V$, and $\hat{v}$ and $O(\exp (-\tau))$ as $\tau \rightarrow \infty$.

In order to satisfy the first inequality of (iii), Theorem 3.1, we require $U$ and $V$ to satisfy the equation

$$
\Delta U+H n_{0} f^{\prime}\left(\theta_{0}\right) U+H V f\left(\theta_{0}\right)=-\exp (-\tau) .
$$

By the principle of superposition we can write

$$
U=\exp (-\tau) A+V B
$$

where

$$
\Delta A+H n_{0} f^{\prime}\left(\theta_{0}\right) A=-1, \quad \Delta B+H n_{0} f^{\prime}\left(\theta_{0}\right) B=-H f\left(\theta_{0}\right), \quad A=B=0 \quad \text { on } \quad \partial D .
$$

By the maximum principle, $A$ and $B$ are positive (see the discussion at the end of the proof). The function $V$ is still to be determined. In order that the second inequality of (iii), Theorem 3.1, be satisfied, we choose $\hat{v}$ and $V$ so that

$$
\Delta(-\hat{v})-(-v) f\left(\theta_{0}\right)-n_{0} f^{\prime}\left(\theta_{0}\right) U-\frac{d}{d \tau}(-v)=\exp (-\tau), \quad \partial \hat{v} / d v=0
$$

Note that in the second inequality of (iii) we must insert the upper temperature (given by $\left.T_{l}+\epsilon^{l} V-\epsilon^{l+1} \hat{v}\right)$. The solvability problem for the above Neumann problem is

$$
\frac{-d V}{d \tau}-V \overline{f\left(\theta_{0}\right)}+n_{0} \overline{f^{\prime}\left(\theta_{0}\right) U}=\exp (-\tau) .
$$

Substituting the expression for $U$ in terms of $V$, we get

$$
\frac{d v}{d \tau}+V \overline{f\left(\theta_{0}\right)}-n_{0} \overline{\left.f^{\prime}\left(\theta_{0}\right) \exp (-\tau) A+V B\right)}=\exp (-\tau) .
$$

We solve this equation for $V$, taking $V(0)=0$. Then, because of the positivity of $A$ and $B$, $v(\tau)>0$ for $\tau>0$. Furthermore, since $\overline{f\left(\theta_{0}\right) \geq 1}, V(\tau)=O(\exp (-\tau))$ as $\tau \rightarrow \infty$. It is now easily seen that $U>0$ and that $U(\tau)=O(\exp (-\tau))$ as $\tau \rightarrow \infty$ as well, so the construction of the functions $U, V$, and $\hat{v}$ is complete.

It remains to prove that $A$ and $B$ are positive. As we observed in Sec. 5, the principle eigenvalue of $\Delta+H n_{0} f^{\prime}\left(\theta_{0}\right)$ is negative. That is, there is a $\lambda_{1}>0$ and a $\varphi \geq 0$ such that

$$
\Delta \varphi+H n_{0} f^{\prime}\left(\theta_{0}\right) \varphi+\lambda_{1} \varphi=0,\left.\quad \varphi\right|_{\partial D}=0 .
$$

Under a small perturbation of the boundary conditions, namely,

$$
\delta(\partial \varphi / \partial v)+\varphi=0 \quad(\delta \text { small and positive }),
$$


we still have a positive principle eigenvalue and eigenfunction. That is, there exist $\lambda>0$ and $\varphi>0$ such that

$$
\Delta \varphi+H n_{0} f^{\prime}\left(\theta_{0}\right) \varphi+\lambda \varphi=0, \quad \delta(\partial \varphi / \partial v)+\varphi=0 .
$$

This modified function $\varphi$ is strictly positive on $\bar{D}$. For, if $\varphi=0$ on $\partial D$, then $\partial \varphi / \partial \nu=0$ at a boundary point where $\varphi$ attains a minimum, and this is impossible by the boundarypoint lemma for elliptic partial differential equations. Therefore $\varphi$ satisfies the inequalities

$$
\begin{gathered}
\Delta \varphi+H n_{0} f^{\prime}\left(\theta_{0}\right) \varphi<0 \text { in } D, \\
\varphi>0 \text { on } \bar{D} .
\end{gathered}
$$

On the other hand, $A$ satisfies the differential inequality

$$
\Delta A+H n_{0} f^{\prime}\left(\theta_{0}\right) A \leq 0 .
$$

We set $A=\varphi W$ and substitute this expression into the above inequality. We obtain

$$
\varphi \Delta w+2(\nabla \varphi) \cdot(\nabla w)+w\left(\Delta \varphi+H n_{0} f^{\prime}\left(\theta_{0}\right) \varphi\right) \leq 0 .
$$

Since the coefficient of $w$ is negative, $w$ cannot attain an interior negative minimum. Since $w=0$ on $\partial D, w$ must be non-negative in the interior of $D$, and so therefore is $A$. The same argument applies to $B$.

7. Perturbation from the metastable state. In this section we construct a uniform approximation to the solution of the initial-value problem (4.1)-(4.3)-that is, an approximation which is valid uniformly on $0<t<\infty$. This approximation is constructed for the case of subcritical combustion.

We consider approximations of the form

$$
\theta=\theta_{0}+w_{0}+\epsilon w, \quad n=n_{0}+\xi_{0}+\epsilon \xi
$$

where $\theta_{0}(x, \tau)$ and $n_{0}(\tau)$ are the first terms of the metastable state constructed in Sec. 5 . Substituting these expressions in Eqs. (4.1)-(4.2), we get

$$
\begin{aligned}
\Delta \theta+H n f(\theta) & -\frac{\partial \theta}{\partial t}=\left(\Delta w_{0}-\frac{\partial w_{0}}{\partial t}+H\left(n_{0}+\xi_{0}\right) f\left(\theta_{0}+w_{0}\right)-H n_{0} f\left(\theta_{0}\right)\right) \\
& +\epsilon\left(\Delta w-\frac{\partial w}{\partial t}+H\left(n_{0}+\xi_{0}\right) f^{\prime}\left(\theta_{0}+w_{0}\right) w+H \xi f\left(\theta_{0}+w_{0}\right)-\frac{\partial \theta_{0}}{\partial \tau}\right)+O\left(\epsilon^{2}\right)
\end{aligned}
$$

and

$$
\begin{aligned}
\Delta n & -\epsilon n f(\theta)-\frac{\partial n}{\partial t} \\
& =\left(\Delta \xi_{0}-\frac{\partial \xi_{0}}{\partial t}\right)+\epsilon\left(\Delta \xi-\frac{\partial \xi}{\partial t}-\epsilon \xi f\left(\theta_{0}+w_{0}\right)-\left(n_{0}+\xi_{0}\right) f\left(\theta_{0}+w_{0}\right)-\frac{d n_{0}}{d \tau}\right)+O\left(\epsilon^{2}\right) .
\end{aligned}
$$

Remark: In the above "expansion" we have grouped the term $\epsilon^{2} \xi f\left(\theta_{0}+w_{0}\right)$ with the other terms of order $\epsilon$. This term, even though it is an order of magnitude smaller, nevertheless provides some dissipation in the expression for $\xi$ which would otherwise not be present. For, since $\partial \xi / \partial \nu=0$ on $\partial D$, the term $\Delta \xi$ actually gives no dissipation. We cannot expand $n$ in a power series in $\epsilon$. If followed strictly, this procedure would lead, at 
order $\epsilon$, to the expression

$$
\Delta \xi_{1}-\partial \xi_{1} / \partial t=d n_{0} / d \tau+\left(n_{0}+\xi_{0}\right) f\left(\theta_{0}+w_{0}\right)
$$

and $\xi_{1}$ would tend, as $t \rightarrow \infty$, to

$$
\int_{0}^{\infty} \int_{D} \frac{d n_{0}}{d \tau}+\left(n_{0}+\xi_{0}\right) f\left(\theta_{0}+w_{0}\right) d x d t
$$

which, though finite, would be non-zero.

To determine $\xi_{0}$ and $w_{0}$ we solve

$$
\begin{gathered}
\Delta \xi_{0}-\partial \xi_{0} / \partial t=0, \quad \xi_{0}(x, 0)=n(x, 0)-\overline{n(x, 0)}, \\
\Delta w_{0}-\frac{\partial w_{0}}{\partial t}+H\left(n_{0}+\xi_{0}\right) f\left(\theta_{0}+w_{0}\right)-H n_{0} f\left(\theta_{0}\right)=0, \\
w_{0}(x, 0)=\theta(x, 0)-\theta_{0}(x, 0) .
\end{gathered}
$$

From the first equation $\xi_{0} \rightarrow 0$ exponentially as $t \rightarrow \infty$. In the second equation, regarding $\tau$ as a parameter, $w_{0}(x, t) \rightarrow 0$ as $t \rightarrow \infty$. (This assumes, of course, that the initial temperature and concentration lie below criticality.) The equilibrium solutions in the equation for $w_{0}$ are given by

$$
\Delta w_{0}+H n_{0}\left(f\left(\theta_{0}+w_{0}\right)-f\left(\theta_{0}\right)\right)=0 .
$$

They are $w_{0}=0, w_{2}\left(x, H n_{0}\right)-w_{1}\left(x, H n_{0}\right)$ and $w_{3}\left(x, H n_{0}\right)-w_{1}\left(x, H n_{0}\right)$ (for fixed $\tau$ ) (see Sec. 4).

Now we construct comparison functions by a method similar to that employed in Sec. 6. Let $\xi_{2}, \xi_{1}, w_{2}$, and $w_{1}$ denote respectively the upper and lower estimates for concentration and temperature. First we solve

$$
\begin{gathered}
\Delta \xi-\frac{\partial \xi}{\partial t}-\epsilon \xi f\left(\theta_{0}+w_{0}\right)-\left(n_{0}+\xi_{0}\right) f\left(\theta+w_{0}\right)-\frac{d n_{0}}{d \tau}=-\delta \exp (-\tau) \\
\xi(x, 0)=\delta .
\end{gathered}
$$

Then $\xi$ is an increasing function of $\delta$. Let $\delta>0$ and take $\xi_{2}=\xi(\delta)$ and $\xi_{1}=\xi(-\delta)$. To get $w_{1}$ and $w_{2}$ we solve

$$
\begin{gathered}
\Delta w-\frac{\partial w}{\partial t}+H\left(n_{0}+\xi_{0}\right) f^{\prime}\left(\theta_{0}+w_{0}\right) w+H \xi(\delta) f\left(\theta_{0}+w_{0}\right)-\frac{\partial \theta_{0}}{\partial \tau}=-\lambda \exp (-\tau), \\
w(x, 0)=\lambda .
\end{gathered}
$$

Denote this solution by $w=w(\lambda, \delta)$. By a maximum principle argument it is easily seen that $w$ is increasing in $\lambda$ and $\delta$. Letting $\lambda>0, \delta>0$ we take $w_{2}=w(\lambda, \delta)$ and $w_{1}=$ $w(-\lambda,-\delta)$. Then the four appropriate comparison functions are

$$
\begin{aligned}
T_{i} & =\theta_{0}+w_{0}+\epsilon w_{i}, & & i=1,2, \\
n_{i} & =n_{0}(\tau)+\xi_{0}+\epsilon \xi_{i}, & i & =1,2, .
\end{aligned}
$$

The form of the approximations indicates clearly that, if the system is not initially in a metastable state - that is, if the concentration is not constant, and the temperature does not satisfy the corresponding steady state temperature equation-then the system 
first adjusts exponentially on a time scale of order 1 to the metastable state, and then decays slowly (as $e^{-t t}$ ) to $\theta=0, n=0$. The rate at which $\xi_{0} \rightarrow 0$ is determined by the second eigenvalue of the Laplacian under the boundary condition $\partial \xi / \partial \nu=\mathbf{0}$.

\section{REFERENCES}

[1] D. A. Frank-Kamenetzky, Diffusion and heat exchange in chemical kinetics, translated by N. Thon, Princeton Univ. Press, Princeton, N. J., 1955

[2] F. Hoppensteadt, On quasilinear parabolic equations with a small parameter, Comm. Pure Applied Math. 24, 17-38 (1971)

[3] - Asymptotic series solutions of some nonlinear parabolic equations with a small parameter Arch. Rat. Mech. Anal. 35 284-298 (1969)

[4] - Properties of solutions of ordinary differential equations with small parameters, Comm. Pure Appl. Math. 24 807-840 (1971)

[5] P. Fife, Singular perturbation problems whose degenerate forms have many solutions, Applicable Analysis 1, 331-358 (1972)

[6] I. M. Gelfand, Some problems in the theory of quasilinear equations, AHS Translations Ser. 2, 29, 295-381 (1963)

[7] G. R. Gavalas, Nonlinear differential equations of chemically reacting systems, Springer, New York (1968)

[8] J. R. Parks, Criticality criteria . . as functions of activation energy and temperature of assembly, J. Chem. Phys. 34, 46-50 (1961)

[9] J. B. Keller, Perturbation theory, Six Lectures presented at MSU, Lansing, Michigan (1968)

[10] S. Parter, Solutions of a differential equation arising in chemical reactor processes, Computer Sciences Dept. Univ. of Wisconsin, Technical Report \#162, January 1973; SIAM J. Appl. Math. 26, 687-716 (1974)

[11] M. H. Protter and H. Weinberger, Maximum principles in differential equations, Prentice-Hall, Englewood Cliffs, (1967)

[12] D. H. Sattinger, Monotone methods in nonlinear elliptic and parabolic boundary value problems, Indiana Univ. Math. Jour. 21, 979-1000 (1972)

[13] D. H. Sattinger, Stability of solutions of nonlinear equations, Math. Anal. Appl. 39, 1-12 (1972)

[14] R. Aris, Introduction to the study of chemical reactors, Prentice-Hall, Englewood Cliffs, 1965 (1st edition)

[15] D. R. Schneider, N. R. Amundson, and R. Aris, On a mechanism for autocatalysis, Chem. Eng. Sci. 27, 895-905 (1972) 